\title{
Hypoxia-inducible Factor 2 $\alpha$ : A Key Player in Tumorigenesis and Metastasis of Pheochromocytoma and Paraganglioma?
}

Authors

Nicole Bechmann1, 2, 3, 4, Graeme Eisenhofer ${ }^{1,2}$

\section{Affiliations}

1 Institute of Clinical Chemistry and Laboratory Medicine, University Hospital Carl Gustav Carus, Medical Faculty Carl Gustav Carus, Technische Universität Dresden, Dresden, Germany

2 Department of Medicine III, University Hospital Carl Gustav Carus, Medical Faculty Carl Gustav Carus, Technische Universität Dresden, Dresden, Germany

3 German Institute of Human Nutrition Potsdam-Rehbruecke, Department of Experimental Diabetology, Nuthetal, Germany

4 German Center for Diabetes Research (DZD), MünchenNeuherberg, Germany

Key words genotype-phenotype relationships, HIF2 $\alpha$ inhibitors, hypoxia, invasion-metastasis cascade, neuroendocrine-tomesenchymal transition

$\begin{array}{ll}\text { received } & 03.05 .2021 \\ \text { revised } & 10.06 .2021 \\ \text { accepted } & 04.06 .2021\end{array}$

published online 28.07 .2021

Bibliography

Exp Clin Endocrinol Diabetes 2022; 130: 282-289

DOI 10.1055/a-1526-5263

ISSN 0947-7349

(c) 2021. Thieme. All rights reserved. Georg Thieme Verlag, Rüdigerstraße 14, 70469 Stuttgart, Germany

\section{Correspondence}

Nicole Bechmann, PhD

University Hospital Carl Gustav Carus, Medical Faculty

Carl Gustav Carus, Technische Universität Dresden,

Fetscherstrasse 74

01307 Dresden

Germany

Tel.: + 49351 458-19687

Nicole.bechmann@uniklinikum-dresden.de

\section{ABSTRACT}

Germline or somatic driver mutations linked to specific phenotypic features are identified in approximately $70 \%$ of all catecholamine-producing pheochromocytomas and paragangliomas (PPGLs). Mutations leading to stabilization of hypoxia-inducible factor $2 \alpha$ (HIF $2 \alpha$ ) and downstream pseudohypoxic signaling are associated with a higher risk of metastatic disease. Patients with metastatic PPGLs have a variable prognosis and treatment options are limited. In most patients with PPGLs, germline mutations lead to the stabilization of HIF2 $\alpha$. Mutations in HIF2 $\alpha$ itself are associated with adrenal pheochromocytomas and/or extra-adrenal paragangliomas and about $30 \%$ of these patients develop metastatic disease; nevertheless, the frequency of these specific mutations is low (1.6-6.2\%). Generally, mutations that lead to stabilization of HIF2 $\alpha$ result in distinct catecholamine phenotype through blockade of glucocorticoid-mediated induction of phenylethanolamine $\mathrm{N}$-methyltransferase, leading to the formation of tumors that lack epinephrine. HIF $2 \alpha$, among other factors, also contributes importantly to the initiation of a motile and invasive phenotype. Specifically, the expression of HIF2 $\alpha$ supports a neuroendocrine-to-mesenchymal transition and the associated invasion-metastasis cascade, which includes the formation of pseudopodia to facilitate penetration into adjacent vasculature. The HIF2 $\alpha$-mediated expression of adhesion and extracellular matrix genes also promotes the establishment of PPGL cells in distant tissues. The involvement of HIF $2 \alpha$ in tumorigenesis and in multiple steps of invasion-metastasis cascade underscores the therapeutic relevance of targeting HIF2 $\alpha$ signaling pathways in PPGLs. However, due to emerging resistance to current HIF2 $\alpha$ inhibitors that target HIF2 $\alpha$ binding to specific partners, alternative HIF2 $\alpha$ signaling pathways and downstream actions should also be considered for therapeutic intervention. 


$\begin{array}{ll}\text { ABBREVIATIONS } \\ \text { ARNT } & \text { aryl hydrocarbon receptor nuclear translocator } \\ \text { ATRX } & \text { ATRX chromatin remodeler } \\ \text { cCRCC } & \text { clear cell renal cell carcinoma } \\ \text { DLST } & \text { dihydrolipoamide S-succinyltransferase } \\ \text { EMT } & \text { epithelial-mesenchymal transition } \\ \text { EPAS1 } & \text { endothelial PAS protein 1 } \\ \text { FH } & \text { fumarate hydratase } \\ \text { FGFR1 } & \text { fibroblast growth factor receptor 1 } \\ \text { GOT2 } & \text { glutamic-oxaloacetic transaminase 2 } \\ \text { HIF2 } \alpha & \text { hypoxia-inducible factor 2 } \alpha \\ \text { HREs } & \text { hypoxia-responsive elements } \\ \text { IDH } & \text { isocitrate dehydrogenases } \\ \text { NF1 } & \text { neurofibromin 1 } \\ \text { MAX } & \text { MYC associated factor } X \\ \text { MDH2 } & \text { malate dehydrogenase 2 } \\ \text { neuroendoMT } & \text { neuroendocrine-to-mesenchymal transition } \\ \text { PNMT } & \text { phenylethanolamine N-methyltransferase } \\ \text { PPGLs } & \text { pheochromocytomas and paragangliomas } \\ \text { PHDs } & \text { prolyl hydroxylases } \\ \text { RET } & \text { Ret proto-oncogene } \\ \text { SDH } & \text { succinate dehydrogenase } \\ \text { SLC25A11 } & \text { mitochondrial 2-oxoglutarate/malate carrier } \\ \text { TET } & \text { ten-eleven translocation } \\ \text { TERT } & \text { telomerase reverse transcriptase } \\ \text { TH } & \text { tyrosine hydroxylase } \\ \text { TMEM127 } & \text { transmembrane protein 127 } \\ \text { VHL } & \text { von Hippel-Lindau } \\ & \end{array}$

\section{Introduction}

Pheochromocytomas and paragangliomas (PPGLs) are rare neural crest-derived tumors (2-8 cases per million per year) that respectively originate from chromaffin cells of the adrenal medulla or the extra-adrenal sympathetic paraganglia [1]. Paragangliomas of the head and neck on the other hand are derived mainly from non-chromaffin cells associated with parasympathetic ganglia. Most PPGLs do not metastasize, but up to $20 \%$ of patients with PPGLs present with distant metastases at sites where chromaffin cells are normally absent, such as lymph nodes, lung, liver, and bones [2-4]. Metastatic disease may be identified at either initial diagnosis (synchronous disease) or only become apparent at follow-up (metachronous disease). This article reviews the role of hypoxia-inducible factor $2 \alpha$ (HIF $2 \alpha$ ), also referred to as endothelial PAS protein 1 (EPAS1), in tumorigenesis and metastasis of PPGLs. In this context, we also highlight the influence of HIF2 $\alpha$ on catecholamine phenotype and its effects on different steps of the invasion-metastasis cascade to clarify the therapeutic relevance of stabilizing an altered HIF2 $\alpha$ for treatment of patients with metastatic PPGLs.

Whether the metastatic disease is synchronous or metachronous, treatment options are limited and the prognosis is poor, though highly variable. An early diagnosis or even prediction of metastatic disease in metachronous cases or patients screened due to hereditary risk may nevertheless be useful for earlier intervention and an improved outcome for any affected patient; to this end, several risk factors have been well established to be associated with metastatic disease. These include germline mutations in succinate dehydrogenase (SDH) subunit B (SDHB), large tumor size, extra-adrenal location, and elevated plasma methoxytyramine concentrations $[2,5,6]$. Telomerase activation and ATRX chromatin remodeler (ATRX) mutations are also described as independent risk factors for metastatic PPGLs [7]. Our recent findings further demonstrate that patients with PPGLs characterized by activation of pseudohypoxic pathways due to an increased expression and stabilization of HIF $2 \alpha$ are also at high risk of metastatic disease [8]. This association also appears to relate to some other established risk factors.

Hypoxia signaling has a far-reaching impact on cellular differentiation and tumorigenesis mainly driven by the two main HIF $\alpha$ isoforms: HIF1 $\alpha$ and HIF2 $\alpha$, which are structurally comparable and mostly have complementary functions $[9,10]$. In presence of oxygen, proline residues within HIF $\alpha$ subunits are hydroxylated by $\alpha$-ketoglutarate-and oxygen-dependent prolyl hydroxylases (PHDs); this then allows for von Hippel-Lindau (VHL) tumor suppressor-mediated proteasomal degradation of the HIF proteins ( Fig. 1a). In absence of oxygen (hypoxia), HIF $\alpha$ subunits form transcriptionally active complexes with aryl hydrocarbon receptor nuclear translocator (ARNT, also known as HIF $\beta$ ) and co-factors (e. g., CREB-binding protein and p300) followed by C-terminal transactivation of genes possessing hypoxia-responsive elements (HREs) ( Fig. 1b). In addition to HRE-mediated mechanisms, both HIF $\alpha$ subunits show interactions with NOTCH, WNT, and MYC pathways, thereby regulating gene expression through these additional pathways [11]. During cellular adaptation to hypoxia, the activity of HIF $\alpha$ differs temporally; while HIF1 $\alpha$ primarily mediates the acute response to severe hypoxia, HIF2 $\alpha$ mediates the response to chronic, even mild hypoxia [12]. This differential regulation is mediated by different hypoxia-associated factors and involves distinct cellular functions [13]. Moreover, several studies have indicated an oncogenic activity of HIF $2 \alpha$, and tumor suppressor role of HIF $1 \alpha$ $[14,15]$. HIF1 $\alpha$ is ubiquitously expressed in many cell types, while HIF2 $\alpha$ expression is restricted to specific cellular populations, including endothelial cells, neural crest cells, and glial cells [16].

\section{HIF2 $\alpha$-dependent genotype-phenotype relationships in PPGLs}

Currently, germline or somatic driver mutations can be identified in approximately $70 \%$ of catecholamine-producing PPGLs $[17,18]$. Close links between genotype and phenotype including the risk of metastatic disease, age of onset, syndromic presentation, and a predominant anatomic site have been recognized [19, 20]. PPGLs are most broadly classified into two main clusters in accordance with their transcriptional profiles [21,22]. Cluster 1 PPGLs comprise those with mutations encoding two groups of genes; these are either directly involved in the stabilization of HIFs or encode components of the tricarboxylic acid (TCA) cycle. The former include VHL, EGLN1/2 (encoding PHD1/2), and HIF2 $\alpha$, whereas the latter include SDH subunits (SDHA, SDHB, SDHC, SDHD), fumarate hydratase $(F H)$, malate dehydrogenase $2(M D H 2)$, mitochondrial 2-oxoglutarate/malate carrier (SLC25A11), isocitrate dehydrogenases 
(IDH1/IDH2/IDH3B), glutamic-oxaloacetic transaminase 2 (GOT2), and dihydrolipoamide S-succinyltransferase (DLST) ( Fig. 1c) [18].

All cluster 1 mutations result in the stabilization of HIF $\alpha$ and lead to activation of hypoxia pathways even in the presence of oxygen (pseudohypoxia) [23]. Cluster 1 PPGLs are more prone to metastasize (18.6\% excluding patients with SDHB mutation) compared to cluster 2 PPGLs (4.3\%) caused by mutations of genes including (Ret protooncogene (RET), neurofibromin 1 (NF1), transmembrane protein 127 (TMEM127), H-Ras (HRAS), fibroblast growth factor receptor 1
(FGFR 1), MYC associated factor X (MAX) that lead to activation of kinase signaling pathways [8]. However, due to mutations in $S D H B$, cluster 1 PPGLs are particularly prone to metastasize (75.6\% in [8]) [24]. Both higher expression and stabilization of HIF2 $\alpha$ are characteristics of cluster 1 compared to cluster 2 PPGLs [21,25, 26] and this seems to contribute to the pro-metastatic behavior of cluster 1 tumors [8, 27].

HIF2 $\alpha$ blocks glucocorticoid-mediated induction of phenylethanolamine $\mathrm{N}$-methyltransferase (PNMT), the enzyme that converts

a

Oxygenated conditions - Normoxia

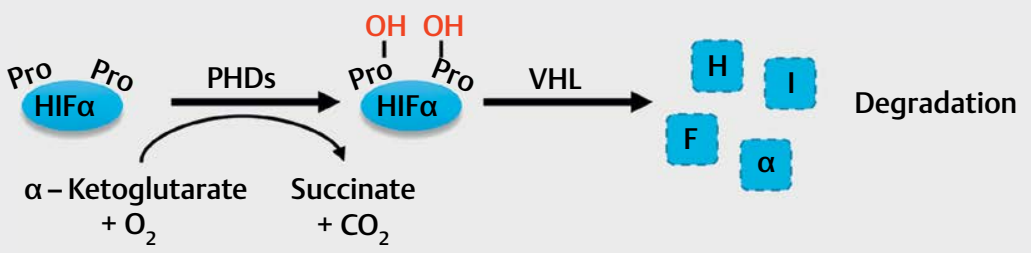

b

\section{Oxygen deficiency - Hypoxia}

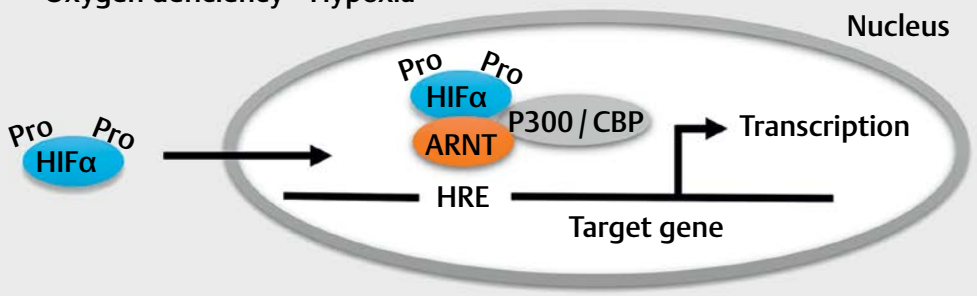

c

Oxygenated conditions - Pseudohypoxia
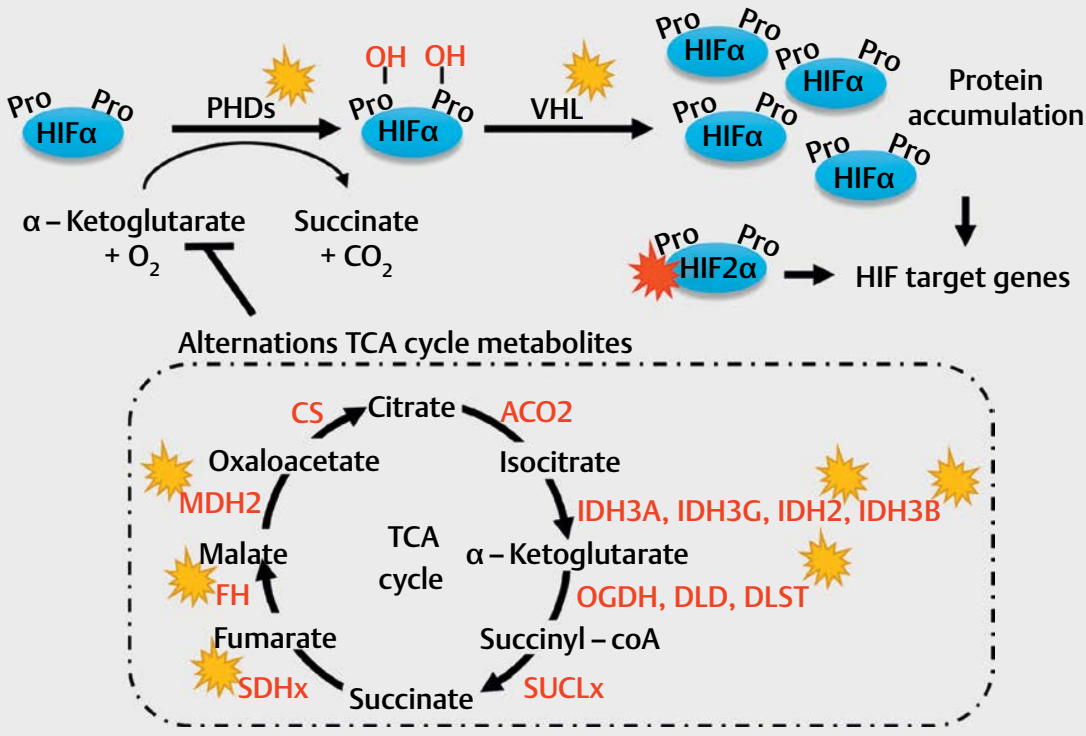

\footnotetext{
- Fig. 1 Oxygen-dependent and independent regulation of HIFa. (a) Under normoxic conditions, HIFa subunits are hydroxylated by $\alpha$-ketoglutarate-and $\mathrm{O}_{2}$-dependent prolyl hydroxylases (PHDs) enabling von Hippel-Lindau (VHL)-mediated degradation of the HIF $\alpha$ protein. (b) Under hypoxic conditions, HIF $\alpha$ subunits form transcriptionally active complexes with ARNT (also known as HIF $\beta$ ) and co-factors followed by C-terminal transactivation of genes possessing hypoxia-responsive elements (HREs). (c) Pseudohypoxic conditions are characterized by increased stabilization of HIF $\alpha$, especially HIF2 $\alpha$, although oxygen is present. In PPGLs, pseudohypoxia can be caused by mutations in hypoxia-related genes encoding PHDs, VHL, and HIF2a themselves, or by mutations in tricarboxylic acid (TCA) cycle-related genes leading to an alteration in TCA cycle metabolites, leading to an inhibition of $\alpha$-ketoglutarate-dependent PHDs. Genes reported as altered in PPGLs are highlighted by stars (yellow: loss-of-function mutation, red: gain-of-function mutation). CBP: CREB-binding protein; FH: fumarate hydratase; MDH2: mitochondrial malate dehydrogenase; CS: citrate synthase; ACO2: mitochondrial aconitase; IDH2: isocitrate dehydrogenase 2; IDH3A/IDH3B/IDH3G: subunits of isocitrate dehydrogenase 3; OGDH/DLD/DLST: subunits of the alpha-ketoglutarate dehydrogenase complex; SUCLx: subunits of succinyl-CoA synthetase; SDHx: subunits of the succinate dehydrogenase complex.
} 
norepinephrine to epinephrine, providing a direct link between genotype and biochemical phenotype [26]. This, therefore, explains the expression of PNMT and epinephrine production by cluster 2 PPGLs, but not by cluster 1 PPGLs. HIF2 $\alpha$ also influences phosphorylation of tyrosine hydroxylase (TH), the rate-limiting step in catecholamine synthesis, thereby affecting cellular dopamine and norepinephrine production [28]. Cluster 1 PPGLs that produce predominantly norepinephrine with varying amounts of dopamine, have lower total tissue catecholamine content than cluster 2 PPGLs; nevertheless, rates of catecholamine secretion and urinary excretion are higher in cluster 1 than cluster 2 PPGLs [29]. This may reflect a more completely developed secretory system in cluster 2 than in cluster 1 PPGLs that acts to restrain otherwise continuous or constitutive secretion [30]. Thus, although cluster 1 tumors tend to secrete catecholamines more actively than cluster 2 tumors, they have lower catecholamine contents per unit of tissue and produce lower amounts of $\mathrm{O}$-methylated metabolites than cluster 2 tumors. The role of HIF $2 \alpha$ in these differences remains unclear.

The predisposing role of HIF2 $\alpha$ expression may also explain the reason for tumorigenesis of certain neural crest derivatives according to mutations that involve stabilization of HIF2 $\alpha$ protein. As mentioned earlier, while HIF1 $\alpha$ is ubiquitously expressed, HIF2 $\alpha$ exhibits a much more restricted expression pattern [16]. In particular, HIF2 $\alpha$ is expressed transiently during the migration of trunk neural crest cells to sympathetic paraganglia and the adrenal medulla, and before further differentiation [31]. This expression impacts migration and proliferation and has also been associated with the development of neural-crest derived sympathoblast precursors to neuroblastoma subtypes with more aggressive and less differentiated features than other subtypes [32]. Furthermore, using single-cell transcriptomics, human embryos were shown to have different neural crest cell derivatives that populate both the sympathetic paraganglia and adrenal medulla and may be responsible for the heterogeneity of associated tumors [33]. It, therefore, seems possible that cluster 1 tumors may be derived from more primitive neural crest derivatives compared to cluster 2 tumors and that this is associated with expression of HIF2 $\alpha$ and different phenotypic features including the propensity for metastasis. Similarly, the younger age of patients with cluster 1 noradrenergic than cluster 2 adrenergic tumors, along with findings of higher proportions of multifocal, extra-adrenal, and metastatic tumors in younger than older patients with PPGLs, has also been proposed to reflect the development of cluster 1 tumors from different populations of chromaffin cell precursors [34, 35].

In line with the above considerations and as outlined by Fliedner et al. [19], the cellular origins and the location of tumors can also influence tumor behavior in terms of catecholamine phenotype and metastatic behavior. The majority of chromaffin cell tumors arise from the adrenal medulla $[35,36]$. About half of these produce norepinephrine exclusively and reflect cluster 1 tumors and the other half, the cluster 2 tumors, present with variable amounts of both norepinephrine and epinephrine [29, 37]. Sympathetic paragangliomas usually exclusively produce norepinephrine, in some cases additional variable amounts of dopamine, and in isolated cases exclusively dopamine [29]. Head and neck paragangliomas, which are derived from parasympathetic ganglia, show only limited expression of TH [38, 39]; thus, most are biochemically non-functional, though about $30 \%$ produce dopamine as manifested by the increase in the dopamine metabolite, methoxytyramine [40].

A recent study focusing on Sino-European differences in the genetic landscape of patients with PPGLs initiated a paradigm shift in the understanding of genotype-phenotype relationships of these genetically heterogeneous tumors [41]. Epinephrine production in the adrenal medulla depends on the expression of PNMT, which is regulated by glucocorticoids produced in the surrounding adrenal cortex [42]. According to previous understanding, mainly based on findings in Caucasian populations, this explains why epinephrine production is confined to adrenal pheochromocytoma [37, 43, 44]. Among the Chinese population, a substantial proportion of extraadrenal PGLs produce epinephrine mainly associated with somatic mutations in HRAS and FGFR1. These findings further clarify that the adrenergic phenotype of PPGLs primarily depends on the underlying genetic mutation rather than tumor location [41].

Nevertheless, the aforementioned revised understanding does not mean that glucocorticoids are not relevant to induction of PNMT, but only that they may act on chromaffin cells to impact phenotypic features more distantly than previously believed. As outlined earlier, failure to induce PNMT, whether at adrenal or extraadrenal locations, is related to the activity of HIF $2 \alpha$ to block glucocorticoid-mediated induction of the enzyme. As also clarified by Qin et al. [26], these activities possibly involve interactions of HIF2 $\alpha$ with the MYC/MAX complex, actions that are independent of binding of HIF $2 \alpha$ to ARNT/HIF $\beta$.

\section{HIF2 $\alpha$ mutations in PPGL tumorigenesis}

Gain-of-function mutations of HIF2 $\alpha$ are associated with pheochromocytomas and extra-adrenal paragangliomas and are often multifocal and recurrent. HIF2 $\alpha$-mutant PPGLs are more frequent in females than in males and are characterized by the production of norepinephrine [23]. Mutations in HIF2 $\alpha$ are predominantly somatic and postzygotic, and are closely located on the oxygen-dependent degradation domain of HIF2 $\alpha$; this hinders hydroxylation by PHDs and degradation by VHL $[45,46]$. Germline mutations (germline variant $\mathrm{F} 374 \mathrm{Y}$ ) are rare, but mosaicism is more common $[47,48]$. Such patients present with PPGL-somatostatinoma-polycythemia syndrome (Pacak-Zhuang syndrome), which occurs exclusively in females $[46,49,50]$.

In Caucasian cohorts, frequency of HIF2 $\alpha$ mutations in PPGLs varies between $1.6-4.6 \%[8,17,45,51]$, which is lower than in a Chinese cohort at $6.2 \%$ [41]. In our cohort, one-third of patients bearing HIF2 $\alpha$ mutations were diagnosed with metastatic disease [8]. This confirmed a previous study in which $29 \%$ of HIF $2 \alpha$ mutant PPGLs showed metastatic disease [52]. The comparatively high metastatic tendency of HIF2 $\alpha$ mutant PPGLs together with the generally increased metastatic risk of cluster 1 PPGLs, characterized by stabilization of HIF $2 \alpha$, further supports the potential role of HIF2 $\alpha$ as a pro-metastatic factor in PPGLs.

\section{HIF2 $\alpha$-driven mesenchymal transition promotes a pro-metastatic phenotype in PPGLs}

During the invasion-metastasis cascade, tumor cells must pass multiple steps to reach distant organs. Acquisition of a motile and in- 
vasive phenotype (epithelial-mesenchymal transition, EMT) is the initiation step of this cascade and involves various changes in gene expression, including genes encoding proteins involved in cell adhesion and extracellular matrix interactions [53]. Due to the nonepithelial origin of chromaffin cells, PPGLs are assumed to undergo a neuroendocrine-to-mesenchymal transition (neuroendoMT) associated with activation of specific signaling pathways [54-56]. In pheochromocytoma cells, SDHB mutation-induced neuroendoMT is associated with a pro-metastatic phenotype [54]. Our data also confirmed the involvement of HIF2 $\alpha$ in this transition. The expression of HIF $2 \alpha$ leads to changes in focal adhesion and extracellular matrix-receptor interaction pathways [8].

In relation to the above-mentioned findings, a study from Morin et al. showed that the neuroendoMT and pro-metastatic phenotype of SDHB-deficient cells results from synergistic effects of HIF2 $\alpha$ and ten-eleven translocation (TET) dioxygenase-mediated hypermethylation [27]. The 2-oxoglutarate-dependent TET hydroxylates DNA-methylated cytosine to form 5-hydroxymethylocystosine and is thereby directly involved in epigenetic regulation. Especially oncometabolite-driven tumors, such as PPGLs of the TCA cycle-related cluster 1 , and here in particular tumors with SDHB mutations show hypermethylation [57, 58]. A genome-wide DNA methylation analysis in metastatic PPGLs identified hypermethylation of negative elongation factor complex member $E$ (RDBP) as a prognostic marker for stratifying patients according to the risk to develop metastatic disease [59]. This, therefore, provides a potential explanation for the much higher metastatic risk in patients with PPGLs due to mutations of SDHB compared to other cluster 1 genes. Thus, although HIF2 $\alpha$ may be pro-metastatic, other factors clearly play important roles in the development of metastatic disease. These factors can include secondary mutations, such as those in ATRX, TERT, and $p 53$, which may occur after initiation of tumorigenesis and lead to an event that further contributes to the development and progression of metastatic disease $[7,60]$.

In addition to its participation in neuroendoMT and thus the initiation of tumor cell migration from a primary tumor, HIF2 $\alpha$ is also involved in the formation of pseudopodia in PPGLs [8]. This critical step of mesenchymal cell migration enables intravasation; this involves penetration by tumor cells of the endothelial basement membrane, thereby facilitating their entry into adjacent blood or lymph vessels [61]. The increased ability of HIF2 $\alpha$-expressing cells to attach to extracellular matrixes, such as laminin, facilitates the

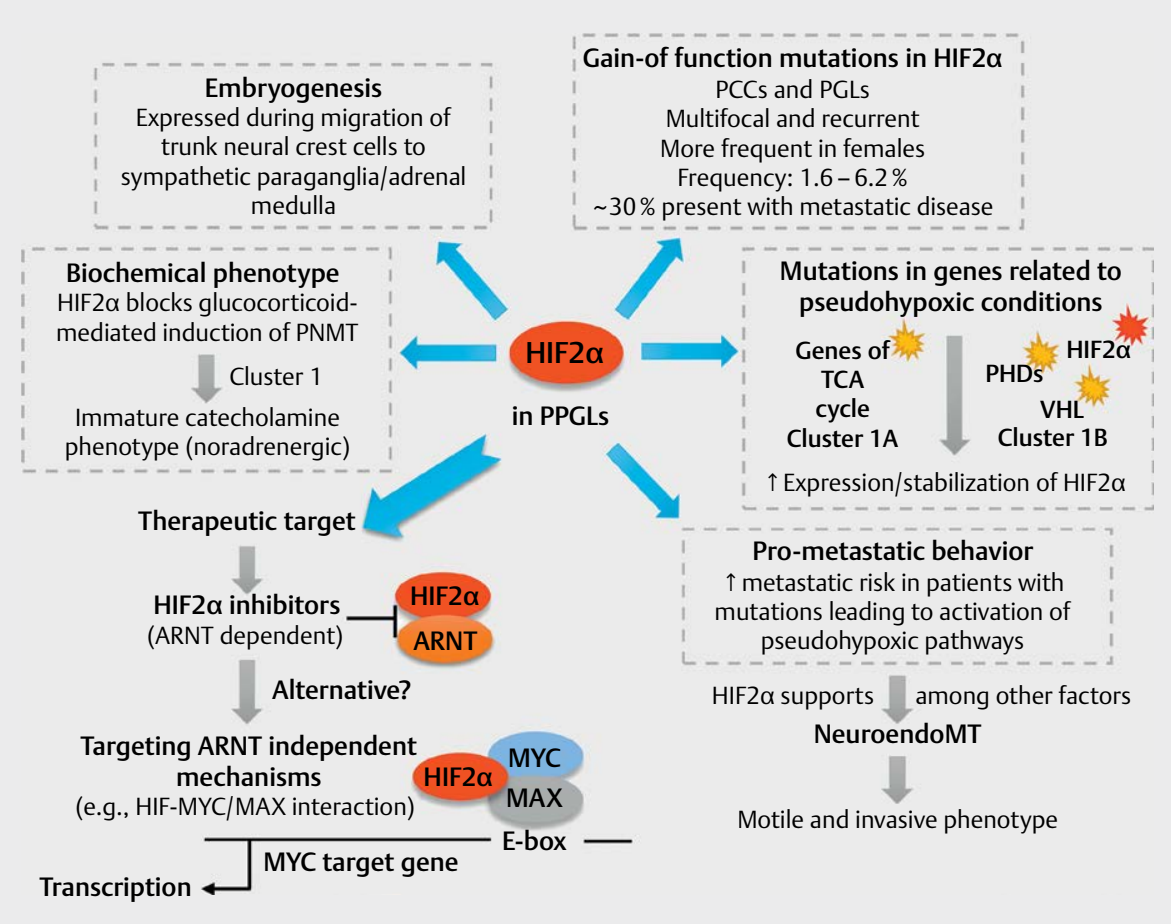

- Fig. 2 Involvement of HIF2 $\alpha$ in tumorigenesis and metastasis of cluster 1 pheochromocytomas and paragangliomas (PPGLs). During embryogenesis, HIF2 $\alpha$ is involved in the migration of trunk neural crest cells to sympathetic paraganglia or adrenal medulla. Mutations in HIF $2 \alpha$ predispose to the development of pheochromocytomas (PCCs) and paragangliomas (PGLs). Moreover, mutations in genes related to activation of hypoxia pathways (pseudohypoxic cluster 1 PPGLs) are characterized by an increased expression and stabilization of HIF2 $\alpha$. Patients with pseudohypoxic cluster 1 PPGLs bear a higher metastatic risk. HIF2 $\alpha$ supports, among other factors, the pro-metastatic behavior of these tumors by the acquisition of a motile and invasive phenotype (neuroendocrine-to-mesenchymal transition, neuroendoMT). HIF2 $\alpha$ blocks the glucocorticoid-mediated induction of phenylethanolamine $\mathrm{N}$-methyltransferase (PNMT) and is thereby directly linked to the immature catecholamine phenotype of cluster 1 PPGL. The versatile involvement of HIF2 $\alpha$ during tumorigenesis and metastasis in PPGLs makes it an ideal target for therapeutic interventions. Available HIF2 $\alpha$ inhibitors block dimerization with ARNT, but some tumors do not seem to respond to these kinds of inhibitors. Addressing ARNT-independent mechanisms of HIF2a, such as interaction with the MYC/MAX complex, maybe an alternative strategy for these resistant tumors. Genes reported as altered in PPGLs are highlighted by stars (yellow: loss-of-function mutation, red: gain-of-function mutation). ARNT: aryl hydrocarbon receptor nuclear translocator; MAX: MYC associated factor X; PHDs: prolyl hydroxylases; VHL: von Hippel-Lindau tumor suppressor. 
adhesion to the endothelial cell layer of the blood vessel, thereby encouraging subsequent extravasation [8]. The expression of genes involved in focal adhesion and the interaction of extracellular matrix-receptor promotes settlement of tumor cells in the pro-metastatic niche; the enhanced proliferation ability of HIF2 $\alpha$-expressing cells allows further metastatic colonization [8]. The multifaceted involvement of HIF2 $\alpha$ in the invasion-metastasis cascade emphasizes the importance of this factor for potential therapeutic approaches in metastatic PPGLs.

\section{Targeting HIF2 $\alpha$ as a therapeutic option for metastatic PPGLs}

For metastatic PPGLs, treatment options are limited and could benefit from personalized considerations according to the nature of underlying disease-causing mutations [62]. The enlarging understanding of the genetic background and associated molecular alterations in metastatic PPGLs offers for the first time a promising approach for individualized treatment of these patients. HIF2 $\alpha$ appears to play an important role in tumorigenesis and metastatic spread of PPGLs ( $\triangleright$ Fig. 2), making it an ideal target for therapeutic approaches. Hypoxia/pseudohypoxia and derived signaling pathways are also associated with increased resistance to chemotherapy and radiotherapy $[63,64]$. Therefore, targeting HIF2 $\alpha$ may provide a potential chemo-and/or radiosensitizing approach to employ alongside other therapies.

Initially, HIF2 $\alpha$ was considered to be undruggable, but the discovery of specific structural features led to the development of small molecule antagonists that can block the dimerization of HIF2 $\alpha$ with ARNT/HIF $\beta[65,66]$. In clear cell renal cell carcinoma (ccRCC), which is frequently characterized by inactivation of $\mathrm{VHL}$ causing an enhanced stabilization of HIF2 $\alpha$, two specific HIF2 $\alpha$ inhibitors, PT2385 and Belzutifan (PT2977) showed promise in phase I clinical trials [67, 68]. Phase II clinical trials in glioblastoma [69] and ccRCC (e.g., NCT03108066, NCT04489771, NCT03401788) are ongoing and a phase I clinical trial is also in planning stages for patients with PPGLs (personal communication). However, some VHL-mutant ccRCC cell lines showed resistance towards HIF2 $\alpha$ inhibitors [70], which is also in line with recent clinical findings [71]. The cCRCC cell lines that were sensitive to HIF2 $\alpha$ inhibitors displayed a distinct HIF2 $\alpha$-dependent gene signature and higher levels of HIF2 $\alpha$ than other cell lines [70]. Resistance can be induced by prolonged treatment with HIF2 $\alpha$ inhibitors $[70,71]$. A gatekeeper mutation in HIF2 $\alpha$ (G323E) that interferes with drug binding further promotes the acquisition of resistance [71]. Our data showed a lack of efficiency of PT2385 in Hif2 $\alpha$-dependent pheochromocytoma cell models [8]. Expression of Hif2 $\alpha$ induced a pro-metastatic phenotype in these cells, which could not be reversed by treatment with PT2385 [8]. Similar results were also obtained in HIF2 $\alpha$ dependent neuroblastomas [72]. This raises the possibility of an ARNT/HIF $\beta$-independent mechanism in these models, for example through interactions with the MYC/MAX complex [73, 74]. These ARNT/HIF $\beta$-independent mechanisms of HIF $2 \alpha$, already mentioned earlier, may offer alternative therapeutic approaches for patients who show resistance to HIF2 $\alpha$ inhibitors ( $>$ Fig. 2).

Further studies are needed to demonstrate the suitability of HIF2 $\alpha$ inhibitors for the treatment of metastatic disease. Due to the described resistance mechanisms and the radio-and chemothera- py resistance associated with hypoxia/pseudohypoxia, suitable combination therapies should also be considered. There is also a need to identify markers that can predict sensitivity towards HIF2 $\alpha$ inhibitors in suitable patients for such therapy.

\section{Conclusion}

Mutations in HIF2 $\alpha$ provide a direct link of HIF2 $\alpha$ to PPGL tumorigenesis. Increased expression and stabilization of HIF2 $\alpha$ are also associated with increased aggressiveness and phenotypic characteristics of these tumors. The involvement of HIF $2 \alpha$ in multiple steps of the invasion-metastasis cascade underscores the therapeutic relevance of targeting impaired HIF $2 \alpha$ signaling pathways in PPGLs. However, because of resistance to current HIF2 $\alpha$ inhibitors that has emerged for certain conditions, ARNT/HIF $\beta$-independent mechanisms of HIF2 $\alpha$ might also be considered for therapeutic intervention.

\section{Acknowledgment}

The work was supported by the Deutsche Forschungsgemeinschaft (DFG, German Research Foundation) within the CRC/Transregio 205, Project No. 314061271 - TRR "The Adrenal: Central Relay in Health and Disease" and the Paradifference Foundation.

\section{Conflict of Interest}

The authors declare that they have no conflict of interest.

\section{References}

[1] Pacak K, Tella SH. Pheochromocytoma and paraganglioma. In: Feingold KR, Anawalt B, Boyce A, et al., Eds. Endotext [Internet]. South Dartmouth (MA): MDText.com, Inc.; 2000

[2] Ayala-Ramirez M, Feng L, Johnson MM et al. Clinical risk factors for malignancy and overall survival in patients with pheochromocytomas and sympathetic paragangliomas: primary tumor size and primary tumor location as prognostic indicators. J Clin Endocrinol Metab 2011; 96: 717-725

[3] Hamidi O, Young WF Jr, Iñiguez-Ariza NM et al. Malignant pheochromocytoma and paraganglioma: 272 patients over 55 years. J Clin Endocrinol Metab 2017; 102: 3296-3305

[4] Fassnacht M, Assie G, Baudin E et al. Adrenocortical carcinomas and malignant phaeochromocytomas: ESMO-EURACAN Clinical Practice Guidelines for diagnosis, treatment and follow-up. Ann Oncol 2020; 31: 1476-1490

[5] Eisenhofer G, Lenders JW, Siegert G et al. Plasma methoxytyramine: a novel biomarker of metastatic pheochromocytoma and paraganglio$\mathrm{ma}$ in relation to established risk factors of tumour size, location and SDHB mutation status. Eur J Cancer 2012; 48: 1739-1749

[6] Eisenhofer G, Deutschbein T, Constantinescu G et al. Plasma metanephrines and prospective prediction of tumor location, size and mutation type in patients with pheochromocytoma and paraganglioma. Clin Chem Lab Med 2020; 59: 353-363

[7] Job S, Draskovic I, Burnichon N et al. Telomerase activation and ATRX mutations are independent risk factors for metastatic pheochromocytoma and paraganglioma. Clin Cancer Res 2019; 25 : 760-770 
[8] Bechmann N, Moskopp ML, Ullrich M et al. HIF2 $\alpha$ supports pro-metastatic behavior in pheochromocytomas/paragangliomas. Endocr-Relat Cancer 2020; 27: 625-640

[9] Hu C-J, Wang L-Y, Chodosh LA et al. Differential roles of hypoxia-inducible factor $1 \alpha(\mathrm{HIF}-1 \alpha)$ and HIF-2 $\alpha$ in hypoxic gene regulation. Mol Cell Biology 2003; 23: 9361-9374

[10] Carroll VA, Ashcroft M. Role of hypoxia-inducible factor (HIF)-1 $\alpha$ versus HIF-2 $\alpha$ in the regulation of HIF target genes in response to hypoxia, insulin-like growth factor-I, or loss of von Hippel-Lindau function: implications for targeting the HIF pathway. Cancer Res 2006; 66: 6264-6270

[11] Kaelin WG Jr, Ratcliffe PJ. Oxygen sensing by metazoans: the central role of the HIF hydroxylase pathway. Molecular Cell 2008; 30: 393-402

[12] Holmquist-Mengelbier L, Fredlund E, Löfstedt T et al. Recruitment of HIF- $1 \alpha$ and HIF- $2 \alpha$ to common target genes is differentially regulated in neuroblastoma: HIF-2 $\alpha$ promotes an aggressive phenotype. Cancer Cell 2006; 10: 413-423

[13] Koh MY, Lemos R, Liu X et al. The hypoxia-associated factor switches cells from HIF-1 $\alpha$-to HIF-2 $\alpha$-dependent signaling promoting stem cell characteristics, aggressive tumor growth and invasion. Cancer Res 2011; 71: 4015-4027

[14] Shen C, Beroukhim R, Schumacher SE et al. Genetic and functional studies implicate HIF1 $\alpha$ as a $14 q$ kidney cancer suppressor gene. Cancer Discov 2011; 1: 222-235

[15] Toledo RA, Qin Y, Srikantan S et al. In vivo and in vitro oncogenic effects of HIF2A mutations in pheochromocytomas and paragangliomas. Endocr-Relat Cancer 2013; 20: 349

[16] Wiesener MS, Jürgensen JS, Rosenberger $C$ et al. Widespread, hypoxia-inducible expression of HIF-2 $\alpha$ in distinct cell populations of different organs. FASEB J 2003; 17: 271-273

[17] Fishbein L, Leshchiner I, Walter $V$ et al. Comprehensive molecular characterization of pheochromocytoma and paraganglioma. Cancer Cell 2017; 31: 181-193

[18] Cascón A, Remacha L, Calsina B et al. Pheochromocytomas and paragangliomas: bypassing cellular respiration. Cancers 2019; 11: 683

[19] Fliedner SM, Brabant G, Lehnert H. Pheochromocytoma and paraganglioma: genotype versus anatomic location as determinants of tumor phenotype. Cell Tissue Res 2018; 372: 347-365

[20] Crona J, Lamarca A, Ghosal S et al. Genotype-phenotype correlations in pheochromocytoma and paraganglioma. Endocr Relat Cancer 2019; 26: $539-550$

[21] Eisenhofer G, Huynh T, Pacak K et al. Distinct gene expression profiles in norepinephrine-and epinephrine-producing hereditary and sporadic pheochromocytomas: activation of hypoxia-driven angiogenic pathways in von Hippel-Lindau syndrome. Endocr-Relat Cancer 2004; 11: 897-911

[22] Dahia PL, Ross KN, Wright ME et al. A HIF1 $\alpha$ regulatory loop links hypoxia and mitochondrial signals in pheochromocytomas. PLoS Genet 2005; 1 : e8

[23] Jochmanova I, Zelinka T, Widimský J Jr et al. HIF signaling pathway in pheochromocytoma and other neuroendocrine tumors. Physiol Res 2014; 63: S251-S262

[24] Gimenez-Roqueplo A-P, Favier ], Rustin P et al. Mutations in the SDHB gene are associated with extra-adrenal and/or malignant phaeochromocytomas. Cancer Res 2003; 63: 5615-5621

[25] Favier J, Plouin P-F, Corvol P et al. Angiogenesis and vascular architecture in pheochromocytomas: distinctive traits in malignant tumors. Am J Pathol 2002; 161: 1235-1246

[26] Qin N, De Cubas AA, Garcia-Martin R et al. Opposing effects of HIF1 $\alpha$ and HIF $2 \alpha$ on chromaffin cell phenotypic features and tumor cell proliferation: Insights from MYC-associated factor X. Int J Cancer 2014; 135: 2054-2064
[27] Morin A, Goncalves ], Moog S et al. TET-mediated hypermethylation primes SDH-deficient cells for HIF2 $\alpha$-driven mesenchymal transition. Cell Rep 2020; 30: 4551-4566. e4557

[28] Bechmann N, Poser I, Seifert $V$ et al. Impact of extrinsic and intrinsic hypoxia on catecholamine biosynthesis in absence or presence of HIF2 $\alpha$ in pheochromocytoma cells. Cancers 2019; 11: 594

[29] Eisenhofer G, Pacak K, Huynh T-T et al. Catecholamine metabolomic and secretory phenotypes in phaeochromocytoma. Endocr-Relat Cancer 2011; 18: 97-111

[30] Eisenhofer G, Huynh T-T, Elkahloun A et al. Differential expression of the regulated catecholamine secretory pathway in different hereditary forms of pheochromocytoma. Am J Physiol-Endocrinol Metab 2008; 295: E1223-E1233

[31] Niklasson CU, Fredlund E, Monni E et al. Hypoxia inducible factor-2a importance for migration, proliferation, and self-renewal of trunk neural crest cells. Dev Dyn 2021; 250: 191-236

[32] Pietras A, Gisselsson D, Øra I et al. High levels of HIF-2 $\alpha$ highlight an immature neural crest-like neuroblastoma cell cohort located in a perivascular niche. J Pathol 2008; 214: 482-488

[33] Kameneva P, Artemov AV, Kastriti ME et al. Single-cell transcriptomics of human embryos identifies multiple sympathoblast lineages with potential implications for neuroblastoma origin. Nat Genet 2021: 1-13

[34] Eisenhofer G, Timmers HJ, Lenders JW et al. Age at diagnosis of pheochromocytoma differs according to catecholamine phenotype and tumor location. J Clin Endocrinol Metab 2011; 96: 375-384

[35] Pamporaki C, Hamplova B, Peitzsch M et al. Characteristics of pediatric vs adult pheochromocytomas and paragangliomas. J Clin Endocrinol Metab 2017; 102: 1122-1132

[36] Castro-Vega LJ, Buffet A, De Cubas AA et al. Germline mutations in FH confer predisposition to malignant pheochromocytomas and paragangliomas. Hum Mol Genet 2014; 23: 2440-2446

[37] Eisenhofer G, Lenders JW, Goldstein DS et al. Pheochromocytoma catecholamine phenotypes and prediction of tumor size and location by use of plasma free metanephrines. Clin Chem 2005; 51: 735-744

[38] Ibanez M, Valderrama-Canales F], Maranillo E et al. Human laryngeal ganglia contain both sympathetic and parasympathetic cell types. Clin Anat 2010; 23: 673-682

[39] Kummer W, Habeck J-O. Chemoreceptor A-fibres in the human carotid body contain tyrosine hydroxylase and neurofilament immunoreactivity. Neuroscience 1992; 47: 713-725

[40] Rao D, Peitzsch M, Prejbisz A et al. Plasma methoxytyramine: clinical utility with metanephrines for diagnosis of pheochromocytoma and paraganglioma. Eur J Endocrinol 2017; 177: 103-113

[41] Jiang J, Zhang J, Pang $Y$ et al. Sino-European differences in the genetic landscape and clinical presentation of pheochromocytoma and paraganglioma. J Clin Endocrinol Metab 2020; 105: 3295-3307

[42] Wurtman R], Axelrod J. Adrenaline synthesis: control by the pituitary gland and adrenal glucocorticoids. Science 1965; 150: 1464-1465

[43] Isobe K, Nakai T, Yashiro T et al. Enhanced expression of mRNA coding for the adrenaline-synthesizing enzyme phenylethanolamine- $\mathrm{N}$-methyl transferase in adrenaline-secreting pheochromocytomas. J Urol 2000; 163: 357-362

[44] Funahashi H, Imai T, Tanaka Y et al. Discrepancy between PNMT presence and relative lack of adrenaline production in extra-adrenal pheochromocytoma. J Surg Oncol 1994; 57: 196-200

[45] Toledo RA, Qin Y, Srikantan S et al. Effects of HIF2A mutations in pheochromocytomas and paragangliomas. Endocr-Relat Cancer 2013; 20: 349-359

[46] Zhuang Z, Yang C, Lorenzo F et al. Somatic HIF2A gain-of-function mutations in paraganglioma with polycythemia. N Engl J Med 2012; 367: 922-930 
[47] Lorenzo FR, Yang C, Fui MNT et al. A novel EPAS1/HIF2A germline mutation in a congenital polycythemia with paraganglioma. J Mol Med 2013; 91: 507-512

[48] Buffet A, Smati S, Mansuy L et al. Mosaicism in HIF2A-related polycythemia-paraganglioma syndrome. J Clin Endocrinol Metab 2014; 99: E369-E373

[49] Pacak K, Jochmanova I, Prodanov T et al. New syndrome of paraganglioma and somatostatinoma associated with polycythemia. J Clin Oncol 2013; 31: 1690-1698

[50] Yang C, Hong CS, Prchal JT et al. Somatic mosaicism of EPAS1 mutations in the syndrome of paraganglioma and somatostatinoma associated with polycythemia. Hum Genome Var 2015; 2: 1-2

[51] Currás-Freixes M, Piñeiro-Yañez E, Montero-Conde C et al. PheoSeq: a targeted next-generation sequencing assay for pheochromocytoma and paraganglioma diagnostics. J Mol Diagn 2017; 19: 575-588

[52] Därr R, Nambuba J, Del Rivero J et al. Novel insights into the polycythemia-paraganglioma-somatostatinoma syndrome. Endocr-Relat Cancer 2016; 23: 899-908

[53] Lamouille S, Xu J, Derynck R. Molecular mechanisms of epithelial-mesenchymal transition. Nat Rev Mol Cell Biol 2014; 15: 178-196

[54] Loriot C, Domingues M, Berger A et al. Deciphering the molecular basis of invasiveness in Sdhb-deficient cells. Oncotarget 2015; 6: 32955-32965

[55] Loriot C, Burnichon N, Gadessaud N et al. Epithelial to mesenchymal transition is activated in metastatic pheochromocytomas and paragangliomas caused by SDHB gene mutations. J Clin Endocrinol Metab 2012; 97: E954-E962

[56] Calsina B, Castro-Vega LJ, Torres-Pérez R et al. Integrative multi-omics analysis identifies a prognostic miRNA signature and a targetable miR-21-3p/TSC2/mTOR axis in metastatic pheochromocytoma/ paraganglioma. Theranostics 2019; 9: 4946-4958

[57] Letouzé E, Martinelli C, Loriot C et al. SDH mutations establish a hypermethylator phenotype in paraganglioma. Cancer Cell 2013; 23: 739-752

[58] Castro-Vega L], Letouzé E, Burnichon N et al. Multi-omics analysis defines core genomic alterations in pheochromocytomas and paragangliomas. Nat Commun 2015; 6: 1-9

[59] De Cubas AA, Korpershoek E, Inglada-Pérez L et al. DNA methylation profiling in pheochromocytoma and paraganglioma reveals diagnostic and prognostic markers. Clin Cancer Res 2015; 21: 3020-3030

[60] Gieldon L, William D, Hackmann K et al. Optimizing genetic workup in pheochromocytoma and paraganglioma by integrating diagnostic and research approaches. Cancers 2019; 11: 809

[61] Shankar J, Messenberg A, Chan J et al. Pseudopodial actin dynamics control epithelial-mesenchymal transition in metastatic cancer cells. Cancer Res 2010; 70: 3780-3790
[62] Lenders JW, Kerstens MN, Laurence A et al. Genetics, diagnosis, management and future directions of research of phaeochromocytoma and paraganglioma: a position statement and consensus of the working group on endocrine hypertension of the european society of hypertension. J Hypertens 2020; 38: 1443-1456

[63] Seifert V, Richter S, Bechmann N et al. HIF2alpha-associated pseudohypoxia promotes radioresistance in pheochromocytoma: insights from 3D models. Cancers 2021; 13: 385

[64] Luo W, Wang Y. Hypoxia mediates tumor malignancy and therapy resistance. Adv Exp Med Biol 2019; 1136: 1-18

[65] Scheuermann TH, Tomchick DR, Machius M et al. Artificial ligand binding within the HIF2 $\alpha$ PAS-B domain of the HIF2 transcription factor. Proc Natl Acad Sci 2009; 106: 450-455

[66] Rogers JL, Bayeh L, Scheuermann TH et al. Development of inhibitors of the PAS-B domain of the HIF- $2 \alpha$ transcription factor. J Med Chem 2013; 56: 1739-1747

[67] Courtney KD, Infante JR, Lam ET et al. Phase I dose-escalation trial of PT2385, a first-in-class hypoxia-inducible factor-2 $\alpha$ antagonist in patients with previously treated advanced clear cell renal cell carcinoma. J Clin Oncol 2018; 36: 867-874

[68] Xu R, Wang K, Rizzi JP et al. 3-[(1 S, 2 S, 3 R)-2, 3-Difluoro-1-hydroxy7-methylsulfonylindan-4-yl] oxy-5-fluorobenzonitrile (PT2977), a hypoxia-inducible factor $2 \alpha$ (HIF-2 $\alpha$ ) inhibitor for the treatment of clear cell renal cell carcinoma. J Med Chem 2019; 62: 6876-6893

[69] Strowd R, Ellingson B, Wen P et al. ACTR-15. Safety and preliminary activity of PT2385, a first-in-class HIF2-alpha inhibitor, planned interim analysis of an open label, single-arm phase II study in patients with recurrent glioblastoma. Neuro-Oncol 2018; 20: vi14-vi14

[70] Chen W, Hill H, Christie A et al. Targeting renal cell carcinoma with a HIF-2 antagonist. Nature 2016; 539: 112-117

[71] Courtney KD, Ma Y, de Leon AD et al. HIF-2 complex dissociation, target inhibition, and acquired resistance with PT2385, a first-in-class HIF-2 inhibitor, in patients with clear cell renal cell carcinoma. Clin Cancer Res 2020; 26: 793-803

[72] Persson CU, von Stedingk K, Fredlund E et al. ARNT-dependent HIF-2 transcriptional activity is not sufficient to regulate downstream target genes in neuroblastoma. Exp Cell Res 2020; 388: 111845

[73] Gordan JD, Bertout JA, Hu C-J et al. HIF-2 $\alpha$ promotes hypoxic cell proliferation by enhancing c-myc transcriptional activity. Cancer Cell 2007; 11: 335-347

[74] Gordan JD, Thompson CB, Simon MCHIF. and c-Myc: sibling rivals for control of cancer cell metabolism and proliferation. Cancer Cell 2007; 12: $108-113$ 